\title{
Robust Estimation of Physical Activity by Adaptively Fusing Multiple Parameters
}

\author{
Timm Hörmann, Peter Christ, Marc Hesse, Ulrich Rückert \\ Cognitronics and Sensor Systems Group \\ CITEC, Bielefeld University 33619 Bielefeld \\ Email: \{thoerman, pchrist, mhesse, rueckert\}@ techfak.uni-bielefeld.de
}

\begin{abstract}
Raising the awareness of being physically active by utilizing wearable body sensors has become a popular research topic. Recent approaches combine physical and physiological information to obtain a precise prediction of a person's physical activity ratio. However, the error in the determination of physical activity due to invalid physiological values that are resulting from underlying signal disturbances, has so far not been considered. We therefore present a robust measure of activity that fuses accelerometer data, heart rate and other personalized features, and is adaptively responding to missing physiological sensor data. To set up the model, we make use of regression analysis (MARS). Our findings indicate the need for considering signal quality when estimating physical activity. The predictive model shows close agreement $\left(R^{2}=0.97\right)$ to the reference from indirect calorimetry, even if the physiological information is partly corrupted.
\end{abstract}

\section{INTRODUCTION}

Today, in western societies, many people suffer from the consequences of a sedentary way of life [1]. Since being physically active is an important factor towards health and well-being [2], estimating a person's total energy expenditure (TEE) has become a research topic of high interest. Yet, physical activity is only one fraction of the TEE, which comprises different components. The energy expenditure that is attributable to physical activity (PAEE), only contributes about $15 \%$ to $30 \%$ of the TEE. The remaining energy is ascribable to the basal metabolic rate (BMR), the thermic effect of food (e.g. digestion) and other factors (e.g. growth) [34]. To obtain comparable values, describing a person's lifestyle, the physical activity ratio (PAR) is one relevant entity. Due to its definition as a multiple of the BMR, PAR provides a feasible approximation of the amount of converted energy due to muscular activity (PAEE).

A well-established method for the laboratory measurement of TEE is the indirect calorimetry. Measuring the oxygen uptake and the amount of produced carbon dioxide by wearing a respiratory mask allows precise calculation of TEE. Although commonly used laboratory methods are the most accurate to measure PAR, their applicability is usually limited to indoor use. For monitoring PAR in everyday life conditions outside of the laboratory, mobile and unobtrusive devices so called wireless body sensors (WBSs) are increasingly used. In comparison to laboratory methods, the use of WBSs shows greater applicability, and is more suitable to elevate PAR during activities of daily life [5].

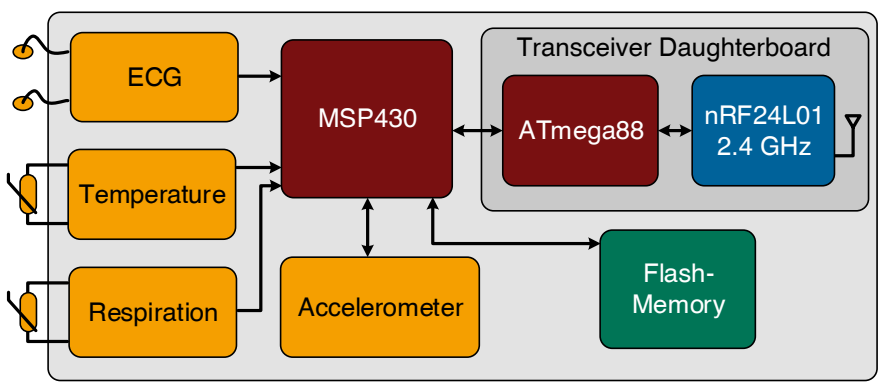

Fig. 1: Technical block diagram of the BG-V4.2 body sensor.

The earliest basic approaches estimated physical activity by measuring the heart rate and using simple linear regression models [6]. In addition accelerometers became popular [7] as they directly measure the physical stress that is more intercomparable than measuring the individual physiological strain of a subject. However, the use of accelerometer signals [8] or heart rate [9] as a single predictive information source is insufficient to estimate PAR. Therefore latest WBSs assess physiological information, like heart rate or respiration rate in addition to the body movement recorded from accelerometer, gyroscope or barometer data. Sensor fusion methods are then used to combine physiological information and body movement. By fusing the various information sources, WBSs are attaining satisfactory results in the prediction of physical activity [10]. An overview about recent methods is given in [11].

We have developed a wireless body sensor (BG-V4.2, fig. 1 that is worn on a chest-strap, and allows to record body movement as well as multiple physiological parameters. While investigating the potential of our WBS to obtain PAR, we noticed that reliable signal quality is a major prerequisite towards an accurate estimation of PAR. Although methods have been presented to identify and eliminate signal disturbances e.g. by advanced filtering methods, obtaining a reliable signal quality might not always be possible, e.g. due to in-band noise, sensor fault or missing compliance of the subjects using the WBS [12 13]. In previously performed experiments [14], we occasionally inspected signal disturbances within the ECG as a result of dry electrodes, motion artifacts or electrical interference, which is the reason why the heart rate becomes invalid, and therefore has to be discarded. 
In this paper, we present an approach for estimating PAR, even if some physiological information need to be rejected. This is achieved by developing a robust measure of activity, based on a multivariate regression approach (MARS, sec. II-F), that allows the modeling of missing sensor data.

The paper is structured as follows: In section II we introduce our WBS, and outline the elicitation of experimental data as well as its following preprocessing. Furthermore, the methods for the quality assessment and the model building process, using the MARS method, are given. Afterwards, the results are presented in section III and subsequently discussed in section IV. Finally, we describe limitations in section V and a conclusion, as well as following implications for further research, are given in section $\mathrm{VI}$.

\section{Methods}

\section{A. Technical Background}

Our WBS (BG-V4.2, fig. 1) enables us to determine a person's heart rate (from recording a 1-lead ECG) and respiration rate (from thoracic movements [15]). Furthermore, it allows recording of the skin temperature and the body accelerations (fig. 1). The accelerometer integrates three sensitive axes and has an adjustable measurement range $( \pm 6 \mathrm{~g}$ to $\pm 24 \mathrm{~g})$ in order to capture accelerations of daily activities as well as sports. Data is stored in a flash memory, and is optionally transmitted through a wireless interface (nRF24L01) to a base station [16]. The whole system is embedded into a single module, which can be used with a conventional chest strap, and is powered by a single CR2025 coin cell. An MSP430FG4618 microcontroller from Texas Instruments is utilized to execute the firmware, and to perform signal processing tasks such as an estimation of the persons walking and running velocity [17].

\section{B. Experimental Design}

To record PAR for different intensities of physical strain, we conducted an experiment on a treadmill that is designed as an incremental exercise test. The treadmill experiment included periods in which the subjects were resting (sitting, standing), as well as periods of walking or running, with a stepwise increase of the velocity $\left(2 \mathrm{~km} \cdot \mathrm{h}^{-1}\right.$ each step) starting from $3 \mathrm{~km} \cdot \mathrm{h}^{-1}$ through to $11 \mathrm{~km} \cdot \mathrm{h}^{-1}$ tbl. I).

In all, 15 healthy subjects (10 male, 5 female) performed the laboratory treadmill experiment, and were asked to provide some personal information tbl. II . All subjects were informed about the experimental procedure, and gave written consent for the participation on the treadmill experiment. To prevent overstraining of the subjects, in 6 cases, running at $11 \mathrm{~km} \cdot \mathrm{h}^{-1}$ was skipped or shortened.

As a reference value for the subjects' TEE, we made use of the indirect calorimetry system "Cortex Metalyzer 3b" 17. As recommended by the manufacturer a calibration of the indirect calorimeter was performed before each test. The Cortex Metalyzer provides the oxygen uptake $\left(\mathrm{VO}_{2}\left[\mathrm{~mL} \cdot \mathrm{min}^{-1}\right]\right)$ and the carbon dioxide production $\left(\mathrm{VCO}_{2}\left[\mathrm{~mL} \cdot \mathrm{min}^{-1}\right]\right)$, as the average

\footnotetext{
${ }^{1}$ Cortex Biophysik GmbH, http://www.cortex-medical.de/
}

TABLE I: Duration and velocities within the different stages of the treadmill incremental exercise test.

\begin{tabular}{lccccccccc}
\hline Stage & 1 & 2 & 3 & 4 & 5 & 6 & 7 & 8 & 9 \\
\hline Description & sit & stand & \multicolumn{8}{c}{ walk / run } & & stand \\
Duration $[\mathrm{min}]$ & 10 & 5 & 3 & 3 & 3 & 3 & 3 & 3 & 10 \\
Velocity $\left[\mathrm{km} \cdot \mathrm{h}^{-1}\right]$ & - & - & 3 & 5 & 7 & 9 & 11 & 5 & - \\
\hline
\end{tabular}

TABLE II: Subject characteristics of learn and test set.

\begin{tabular}{lcccc}
\hline Experiment & age [a] & height $[\mathrm{m}]$ & weight $[\mathrm{kg}]$ & BMI $\left[\mathrm{kg} \cdot \mathrm{m}^{-2}\right]$ \\
\hline learn $(\mathrm{n}=15)$ & $26.2 \pm 3.1$ & $1.76 \pm 0.09$ & $72.9 \pm 14.0$ & $23.2 \pm 2.8$ \\
test $(\mathrm{n}=5)$ & $26.0 \pm 3.5$ & $1.83 \pm 0.07$ & $74.8 \pm 13.1$ & $22.2 \pm 2.4$ \\
\hline
\end{tabular}

of $10 \mathrm{~s}$ epochs. The output can be utilized to calculate the TEE as $\mathrm{kJ} \cdot \mathrm{min}^{-1}$ (eqn. 1, [18]).

$$
\mathrm{TEE}=\left(\left(1.1 \cdot \frac{\mathrm{VCO}_{2}}{\mathrm{VO}_{2}}\right)+3.9\right) \cdot \mathrm{VO}_{2} \cdot 4.1868
$$

The subjects were simultaneously monitored by our WBS. We recorded ECG, heart rate, respiration curve, respiration rate and the upper body movements ( $\mathrm{x}-, \mathrm{y}-, \mathrm{z}$-axis). All signals were measured with a sample rate of $100 \mathrm{~Hz}$. The data was stored in the internal flash memory of the WBS.

\section{Data Set and Data Processing}

The data obtained from the treadmill experiment was used as learning data set. We used an additional data set from a previously performed experiment as a test set, on which 5 subjects (4 male, 1 female) participated [15]. The design was identical to the here described experiment, but it did not cover the initial sitting phase.

To reduce noise, and hence to obtain plausible data (nonpathological) from our WBS, we performed the following preprocessing steps: A fifth order low-pass filter with a cut-off frequency of $20 \mathrm{~Hz}$ was applied to the accelerometer data (x-,y- and z-axis). Similarly a low-pass filter of $12 \mathrm{~Hz}$ and first order was applied to the respiration sensor signal. The recorded ECG was band-pass filtered in the range of $1.6 \mathrm{~Hz}$ to $33.9 \mathrm{~Hz}$.

Since we were interested in predicting physical activity, we needed to convert the measured TEE from kJ to PAR. For this purpose, the BMR for each subject was approximated. The approximation is based on the measured energy expenditure of the first stage of the treadmill experiment, while the subjects were sitting (learning set) or standing (testing set). Therefore, the mean value of the measured TEE, during the last $5 \mathrm{~min}$ of the first stage, was calculated. The value was then divided by the factor 1.2 or 1.4 , respectively (typical PAR for sitting or standing [3]).

\section{Feature extraction}

For feature extraction, we calculated the mean value of the heart rate $\left(\mathrm{HR}\left[\mathrm{min}^{-1}\right]\right)$, respiration rate $\left(\mathrm{RR}\left[\mathrm{min}^{-1}\right]\right)$ and the variance and mean value of the acceleration (ACC $[\mathrm{g}]$ ). We considered acceleration on the $\mathrm{x}$ - (mediolateral) and $\mathrm{z}$-axis 
(anteroposterior) as well as the vertical (eqn. 2), horizontal eqn. 3) and total acceleration vector eqn. 42 In order to match with the target value (PAR) obtained from the indirect calorimetry, each feature was generated for time windows of $10 \mathrm{~s}$ length.

$$
\begin{aligned}
& \mathrm{ACC}_{\mathrm{H}}=\sqrt{a_{y}^{2}} \\
& \mathrm{ACC}_{\mathrm{V}}=\sqrt{a_{x}^{2}+a_{z}^{2}} \\
& \mathrm{ACC}_{\mathrm{C}}=\sqrt{a_{x}^{2}+a_{y}^{2}+a_{z}^{2}}
\end{aligned}
$$

We additionally referred to the subjects' weight (W), height (H) and body mass index (BMI). We chose a small set of basic and commonly known features, as we wanted to create a model, which does not use cardiorespiratory fitness parameters such as resting heart rate, as these values are typically unknown.

\section{E. Quality assessment}

With the intent to design a predictive model that adaptively responds to disturbed sensor signals, we examined the obtained data from our WBS to locate and label time periods containing severe signal disturbances. For the respiration rate, a reference value could be obtained from the indirect calorimetry system. We defined the signal quality of our respiration sensor as disturbed, for periods of time in which the calculated deviation between both measured values exceeds $5 \mathrm{~min}^{-1}$. Since there was no reference information for the ECG signal we manually examined and annotated the ECG data as acceptable or disturbed (fig. 2).

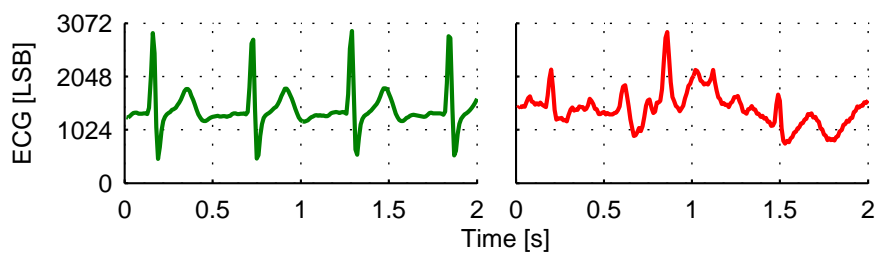

Fig. 2: Example of accepted (green) and rejected ECG (red).

The annotated data sets were used to implement two basic classifiers. To do so, we followed the suggestions from [12], on how to estimate the noise level of the ECG. As a result, we derived two minimal decision tree based classifiers to automatically detect disturbances, in both ECG and respiration sensor signal.

\section{F. Machine Learning and Model Selection}

To establish a model that predicts PAR, the method of multivariate adaptive regression splines (MARS) was chosen, as it has shown good results in predicting physical activity [19]. A MARS model can be written as a weighted $(\beta)$ sum of multiple linear regressions (eqn. 5), so called

\footnotetext{
${ }^{2}$ Accelerometry features are written as $\mathrm{ACC}_{\{\mathrm{X}|\mathrm{Z}| \mathrm{H}|\mathrm{V}| \mathrm{C}\}\{\mathrm{M} \mid \mathrm{V}\}}$ where $\mathrm{X}, \mathrm{Z}, \mathrm{H}, \mathrm{V}, \mathrm{C}$ defines the corresponding axis or direction (horizontal, vertical, total) and $\mathrm{M}$ or $\mathrm{V}$ indicates mean or variance value, respectively.
}

basis functions $(\mathrm{BF}, h)$. Each $\mathrm{BF}$ is build up as a linear function, which describes a local fraction, of one specific independent variable $(x)$, out of the total feature space $(X)$. Through interactions between various BF, a MARS model is suited to map complex, non-linear relationships, between the independent and the dependent variable (target, $\hat{Y}$ ). Hence MARS is offering the advantages of machine learning methods like neural nets, in modeling non-linear and high dimensional relations. Yet, a MARS equation is implemented as easily and resource efficiently as multiple linear regression, because it only consists of linear functions [20]. To train a MARS model we made use of the Salford Predictive Modeler suite 3

$$
\begin{gathered}
\hat{Y}(X)=\beta_{0}+\sum_{m=1}^{M} \beta_{m} \cdot h_{m}(x \in X) \\
h_{m}=\max (0, x-c) \text { or } \max (0, c-x) \text { or } h_{n} \cdot h_{o} \\
: c=\text { const. }, n \neq o
\end{gathered}
$$

The subjects' PAR was chosen as target variable $y \in$ $Y=y_{1}, \ldots, y_{n}$. The prediction (target variable) is thereby calculated from the feature vector $x \in X=x_{1}, \ldots, x_{n}$, which consists of heart rate (HR), respiration rate (RR), the accelerometry features (sec. II-C) as well as of the subjects characteristics weight $(\mathrm{W})$, height $(\mathrm{H})$ and body mass index (BMI). In addition, we considered signal quality of the physiological values. The features $\mathrm{HR}$ and $\mathrm{RR}$ were marked as missing, in case the underlying signal (ECG or respiration sensor signal) was discarded due to signal disturbances.

In order to prevent overfitting, we selected the model with the lowest MSE on the test set. Furthermore, the prediction is set to $1 \mathrm{PAR}$ for responses below this value to suppress pathological outcome.

Additionally, to evaluate the model's predictive performance, we referred to the mean squared error (MSE, eqn. 6) as a value of precision. Furthermore, we discussed the agreement between indirect calorimetry and the MARS model by the coefficient of determination $\left(\mathrm{R}^{2}\right.$, eqn. 7 ).

$$
\begin{gathered}
\mathrm{MSE}=\frac{1}{n} \sum_{i=1}^{n}\left(\hat{Y}_{i}-Y_{i}\right)^{2} \\
\mathrm{R}^{2}=1-\frac{\operatorname{MSE}(Y, \hat{Y})}{\operatorname{Var}(Y)}
\end{gathered}
$$

\section{RESULTS}

\section{A. Model analysis}

To obtain comparable predictions of physical activity, we converted the measured TEE from $\mathrm{kJ}$ to PAR, by dividing the TEE by the previously approximated BMR. Hereby, we expected to reduce inter-individual variations in the TEE, influenced by the differences in the BMR (e.g. due to distinctions in weight or gender). Indeed, while the variation coefficient between all observations of the TEE has a value of $28.76 \%$,

\footnotetext{
${ }^{3}$ SPM v7.0, http://www.salford-systems.com/products/spm
} 
a)

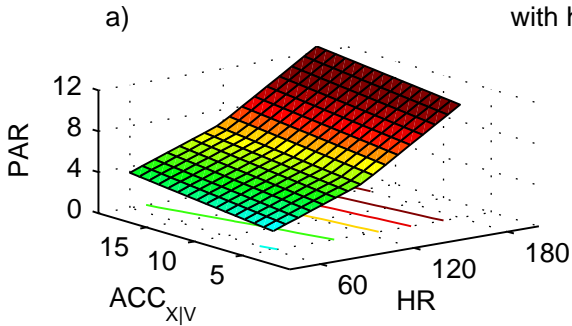

b)

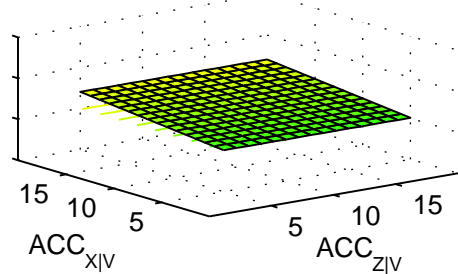

d)

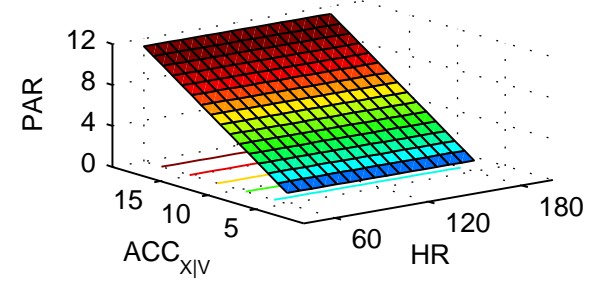

without heart rate

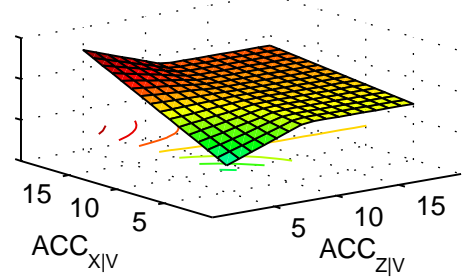

e) model equation

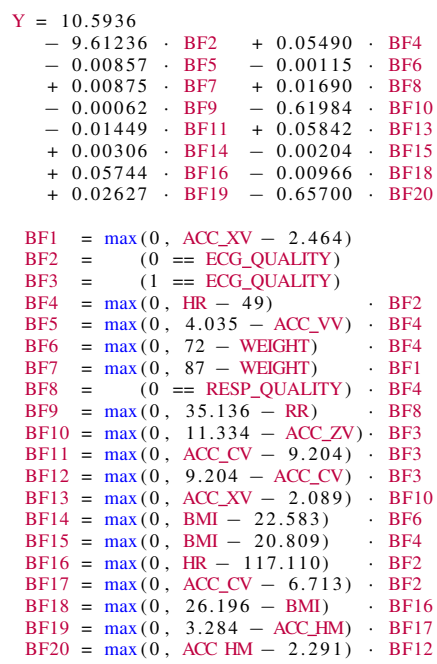

Fig. 3: Prediction response of the trained MARS model (a-d) for various accelerometry features (ACC) and heart rate (HR) both for the cases of accepted $(\mathrm{a} / \mathrm{b})$ and rejected $(\mathrm{c} / \mathrm{d})$ heart rate information. Additionally, the model's equation is given (e).

for PAR this value drops to $8.84 \%$. We infer that through this conversion a normalization of the target variable was achieved.

To sketch the model's response, we illustrated its behavior for the independent variables $\mathrm{ACC}_{\mathrm{X} \mid \mathrm{V}}$ versus $\mathrm{HR}$ and $\mathrm{ACC}_{\mathrm{X} \mid \mathrm{V}}$ versus $\mathrm{ACC}_{\mathrm{Z} \mid \mathrm{V}}($ fig. $3 \mathrm{a} / \mathrm{b}$ ). The remaining variables are fixed The visual analysis indicates that a relation between high heart rate and horizontal acceleration to an increased physical activity ratio has been learned.

Following the design of the experiment, various phases can be identified in the model's response (fig. 3a). While the subject is sitting or standing, a low heart rate in combination with no acceleration is measured, which accordingly results in a low physical activity response. When the subject starts walking or running, the measured acceleration is increased, which leads to a higher physical activity prediction. As soon as the heart rate also starts rising, the model's outcome correspondingly increases. At the end of the experiment the subject is resting in a standing position, and its heart rate slowly decreases. Despite the exercise has ended, the subject is still physically strained. Therefore, during the recovery, the TEE is measurably raised, which is known as excess postexercise oxygen consumption [18]. The model's response covers this event by predicting an increased PAR from a high heart rate, even if no, or practically any, movements are measured. Furthermore, we found that the model's response is discontinuous at a heart rate of $117 \mathrm{~min}^{-1}$. This observation might be explained by the heart rate deflection point that is often found during incremental exercise tests (sec. II-B), as it was discussed in previous work in [21].

As a central finding, heart rate is contributing stronger to the prediction than the accelerometry feature (fig. 3 $)$. However, this result only applies as long as the heart rate is available.

\footnotetext{
${ }^{4} \mathrm{HR}=100, \mathrm{RR}=20$, WEIGHT $=75$, HEIGHT $=1.75, \mathrm{BMI}=24.5$ $\mathrm{ACC}_{\mathrm{XV}}=2.5, \mathrm{ACC}_{\mathrm{ZV}}=1.5, \mathrm{ACC}_{\mathrm{VV}}=30, \mathrm{ACC}_{\mathrm{CV}}=5.5, \mathrm{ACC}_{\mathrm{HM}}=4.5$
}

Otherwise, in case the heart rate information is discarded (due to signal disturbances fig. 3/d), the model's response adapts by changing the weights for the accelerometry features. Thus heart rate information is completely replaced by the information from the accelerometry features (fig. 3f). Quality information of the heart rate thereby not only results in the replacement of the heart rate feature, but the interactions between the various accelerometry features becomes more complex. Moreover, a previously unconsidered accelerometry feature $\left(\mathrm{ACC}_{\mathrm{Z} \mid \mathrm{V}}\right)$ is then included (fig. 3/d).

As can be seen from the models equation (fig. 3 ), a link between respiration and heart rate exists. We found that the respiration rate is lowering the response function as long as the respiration rate is below $35 \mathrm{~min}^{-1}$.

Additionally, interactions among BMI, weight, heart rate and vertical acceleration variance was learned. We found that the prediction of PAR is increased in respect to the subject's weight, height and BMI. Thus, these features are acting as an intercept parameter to the model's response.

\section{B. Prediction Performance}

Overall, the used data sets contain 3667 (learn set) and 715 (test set) observations, of which in 227 (6.2\%) and $93(13.0 \%)$ cases the heart rate was marked as missing.

On the learn set, the model's prediction and the reference PAR from indirect calorimetry are in good agreement $\left(R^{2}=0.94\right.$, MSE $=0.59$ PAR $)$. In addition, including respiration rate quality information is not changing the agreement. However, considering the heart rate quality information increases the agreement $\left(R^{2}=0.97\right)$ and the precision $(\mathrm{MSE}=0.32 \mathrm{PAR})$ of the prediction.

The results of the test set are given in fig. 4. The estimation of PAR by the established model (green) is close to the ground truth obtained from indirect calorimetry (blue). The model's 
Sub. 1

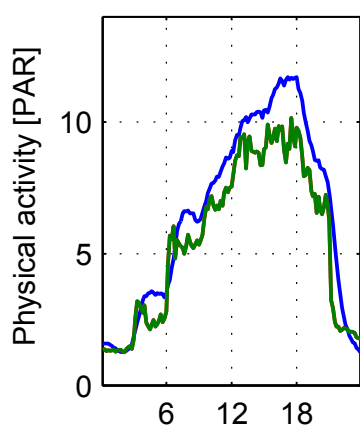

Sub. 2

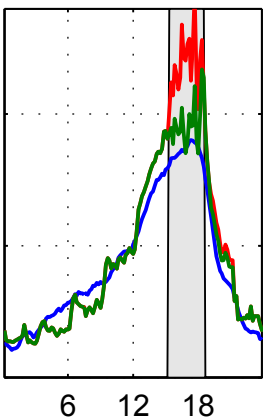

Sub. 3

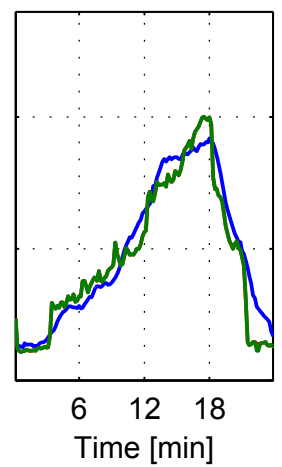

Sub. 4

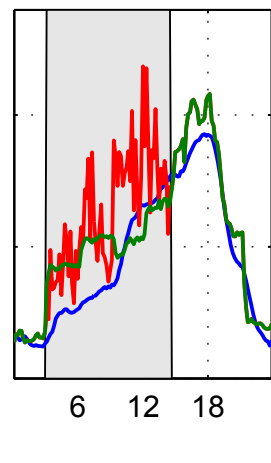

Sub. 5

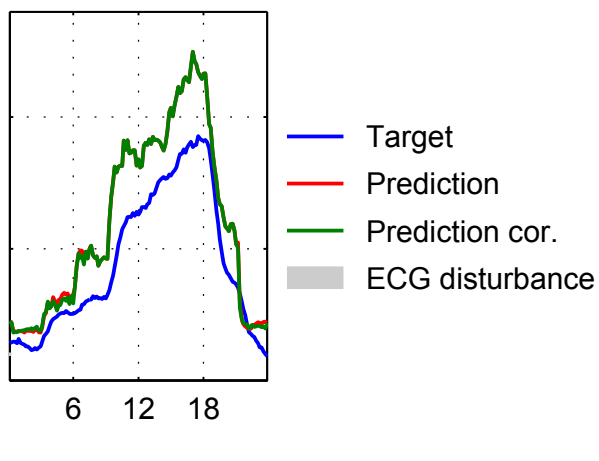

Fig. 4: Results of the PAR prediction made by the MARS model, on the test set data.

agreement $\left(\mathrm{R}^{2}\right)$ reaches $74 \%(\mathrm{MSE}=2.22)$. Again, the estimation is unaffected, if only the quality of the respiration rate's determination is considered. Nevertheless, taking the results of subject 2 and 4 as an example, the consideration of ECG signal disturbances (marked gray), improves the estimation of PAR, in contrast to the uncorrected estimation (red). The model's agreement increases to $82 \%$. Moreover, the precision is improved in case the quality information for the ECG is included (MSE $=1.54)$.

The erroneous determination of the heart rate did partly cause an overestimation of the PAR prediction, as can be seen with reference to the example of subject 2 . This error is successfully compensated through the adaptive response of the model. For subject 4 the overall prediction is also enhanced. However, during light intensity (minute 3 to 9 , walking $3 \mathrm{~km} \cdot \mathrm{s}^{-1}$ to $5 \mathrm{~km} \cdot \mathrm{s}^{-1}$ ) the prediction for subject 4 is smoothed, but also slightly overestimated, in comparison to the ground truth. Yet, during increased intensity walk (minute 9 to 15 , walking $7 \mathrm{~km} \cdot \mathrm{s}^{-1}$ to $9 \mathrm{~km} \cdot \mathrm{s}^{-1}$ ), the estimation's precision is improved as a result of the rejection of invalid heart rate.

Discarding heart and respiration rate (valid and invalid information) leads to a loss of precision. This applies to both, the learn set $\left(\mathrm{R}^{2}=0.70, \mathrm{MSE}=2.98\right)$ and the test set $\left(\mathrm{R}^{2}=0.50, \mathrm{MSE}=4.26\right)$. If only the respiration rate is missing, the predictive performance of the model is slightly affected on the learn set $\left(\mathrm{R}^{2}=0.93\right.$, MSE $\left.=0.72\right)$ and almost unaffected on the test set $\left(\mathrm{R}^{2}=0.82, \mathrm{MSE}=1.57\right)$. As it was shown in the analytic results (sec. III-A), the contribution of the respiration rate to the prediction is limited, but still, slightly lowers the model's outcome. For some subjects this prevents overestimation (subject 3,5), but on the other hand the PAR is (partly) underestimated for the remaining subjects. For this reason, we found the mean error shifted from -0.26 PAR to 0.17 PAR.

In summary, the model provides a proper estimation of the total PAR on the unlearned test data. The mean deviation of the summarized physical activity, between the prediction and the ground truth from indirect calorimetry, is $7.2 \pm 18.6 \%$. Minimum (-4.5\%) and maximum deviation $(33.7 \%)$ was found with subjects 3 or 5 , respectively.

\section{DISCUSSION}

The learned MARS model shows close agreement to the laboratory reference, even on the unlearned test set data $\left(R^{2}=0.82\right)$. Due to the fact that the MARS model just consists of a set of linear equations, and we are utilizing only a basic feature set, the model has minimal computational cost. This allows us to utilize it for on-line prediction of PAR, as it can be directly executed on the microcontroller of our WBS.

While respiration rate only showed little effect, we found that the heart rate information has a stronger influence on the prediction of PAR. Furthermore, we found conflicting results in the test set concerning the respiration rate. A diversity in respiration rate response during physical exercise among various subjects was also discussed in previous work [22]. We therefore deduce, that respiration rate is less crucial to predict PAR, in comparison to heart rate. However, for some subjects within our test set, it prevents over or underestimation of physical activity. Contrary, discarding the heart rate information noticeable reduces accuracy of the prediction. Accordingly, the presented model is showing best results when accelerometry features and heart rate are used in combination.

We demonstrated that the learned model is robust towards signal disturbances, concerning the heart rate and the respiration rate. This is achieved by adjusting weights for the feature set with regard to the availability of the heart and respiration rate features. We found that discarding disturbed heart rate information clearly improves the prediction through preventing the mapping of the fluctuation from heart rate to the estimation of PAR. This is realized by dropping heart rate information, and adjusting the weights of the extracted accelerometry features. Therefore, we conclude that the model's ability to exploit quality information improves the prediction, but it is important that the heart rate information is truly invalid. Otherwise, the combination of the heart rate and the accelerometry features may conceivably be preferred. For this reason, it might be questionable to discard the heart rate if the ECG signal is disturbed, but the calculated heart rate is still feasible. For example, this could be the case for short time periods of signal disturbances within the ECG. It is also conceivable that in these cases, applying a median filter on the 
heart rate, will lead to an improved prediction. However, if the heart rate information is lost, and not usable for a longer time period, e.g. due to a sensor's fault, discarding heart rate seems substantiated. Instead, relaying on the disturbed sensor signal, provokes deviations. Therefore, considering signal quality is mandatory to design a robust regression model that precisely predicts the physical activity ratio.

\section{Limitations}

To the best of our knowledge sensor quality information has never been considered in predictions that estimate energy expenditure or physical activity. However, the results are limited to the conducted treadmill experiment. Furthermore, the model was evaluated on a relatively small test group that only contained young healthy subjects. Thus, for future work it is important to verify our results with a model based on the data of a larger test group.

\section{CONCLUSION}

We implemented a model to predict physical activity from both physical stress (acceleration) and physiological strain (heart rate, respiration rate). We conducted a treadmill experiment on which 15 healthy subjects participated. During the experiment data was obtained by a WBS using indirect calorimetry as ground truth. The model was designed by use of regression analysis (MARS). As a particular extension our model exploits knowledge about the signal quality of the measured physiological values (heart and respiration rate). Thereby, we have shown the necessity for an adaptive estimation that considers sensor value validity.

As we have demonstrated by example, physiological information may provoke an erroneous prediction, if the underlying signal is invalid. We conclude, that a robust measure, which utilizes signal quality information, enables suppression of this error source, without relinquishing the advantages of the combination of physical and physiological information. For this reason, we recommend future approaches focusing on the estimation of physical activity, to consider the signal quality of the recorded physiological values, in order to ensure best predictive performance.

\section{ACKNOWLEDGMENT}

This research was supported by the DFG CoE 277: Cognitive Interaction Technology (CITEC), the German Federal Ministry of Education and Research (BMBF) within the project "KogniHome" and the PhD program "Design of Flexible Work Environments - Human-Centric Use of CyberPhysical Systems in Industry 4.0" supported by the North Rhine-Westphalian funding scheme "Fortschrittskolleg".

The authors are responsible for the contents of this publication. The authors would like to thank Dr. Christian Vobejda from the Department of Sport Science at Bielefeld University for providing access to their sports medicine research lab.

\section{REFERENCES}

[1] M. S. Tremblay, R. C. Colley, T. J. Saunders, G. N. Healy, and N. Owen, "Physiological and health implications of a sedentary lifestyle," Appl Physiol Nutr Metab, vol. 35, no. 6, pp. 725-740, 2010.

[2] US Department of Health and Human Services, Ed., Physical Activity Guidelines for Americans; Be Active, Healthy, and Happy! Office of Disease Prevention \& Health Promotion, 2008.

[3] World Health Organization, Ed., Energy and protein requirements: Report of a Joint FAO/WHO/UNU Expert Consultation. Geneva: World Health Organization, 1985.

[4] C. D. Berdanier, E. B. Feldman, and J. Dwyer, Handbook of Nutrition and Food, Second Edition. Boca Raton: Taylor \& Francis, 2007.

[5] C. Müller, C. Winter, and D. Rosenbaum, "Current objective techniques for physical activity assessment in comparison with subjective methods," Deutsche Zeitschrift fur Sportmedizin, vol. 61, no. 1, p. 11, 2010.

[6] G. Spurr, A. Prentice, P. Murgatroyd, G. Goldberg, J. Reina et al., "Energy expenditure from minute-by-minute heart-rate recording: comparison with indirect calorimetry." Am J Clin Nutr, vol. 48, no. 3, pp. 552-559, 1988.

[7] K. Y. Chen and M. Sun, "Improving energy expenditure estimation by using a triaxial accelerometer," J Appl Physiol, vol. 83, no. 6, pp. 21122122, 1997.

[8] K. Lyden, S. L. Kozey, J. W. Staudenmeyer, and P. S. Freedson, "A comprehensive evaluation of commonly used accelerometer energy expenditure and met prediction equations," Eur J Appl Physiol, vol. 111, no. 2, pp. 187-201, 2011.

[9] M. Altini, J. Penders, R. Vullers, and O. Amft, "Combining wearable accelerometer and physiological data for activity and energy expenditure estimation," in Proc. of the 4th Conf. on Wireless Health, 2013.

[10] S. Brage, N. Brage, P. W. Franks, U. Ekelund, M.-Y. Wong et al., "Branched equation modeling of simultaneous accelerometry and heart rate monitoring improves estimate of directly measured physical activity energy expenditure," J Appl Physiol, vol. 96, no. 1, pp. 343-351, 2004.

[11] S. Liu, R. Gao, and P. Freedson, "Computational methods for estimating energy expenditure in human physical activities," Med Sci Sports Exerc, vol. 44, no. 11, pp. 2138-2146, 112012.

[12] Q. Li, R. G. Mark, and G. D. Clifford, "Robust heart rate estimation from multiple asynchronous noisy sources using signal quality indices and a kalman filter," Physiol Meas, vol. 29, no. 1, pp. 15-32, 12008.

[13] J. Nikolic-Popovic and R. Goubran, "Impact of motion artifacts on heart rate variability measurements and classification performance," in Proc. IEEE Int. Symp. Med. Meas. Appl., 2013, pp. 156-159.

[14] P. Christ and U. Rückert, Identification of Athletes During Walking and Jogging Based on Gait and Electrocardiographic Patterns, ser Biomedical Engineering Systems and Technologies. Springer, 2014, vol. 452, pp. 240-257.

[15] M. Hesse, P. Christ, T. Hörmann, and U. Rückert, "A respiration sensor for a chest-strap based wireless body sensor," in IEEE Sensors, 92014 , pp. 490-493.

[16] P. Christ, B. Neuwinger, F. Werner, and U. Rückert, "Performance analysis of the nRF24L01 ultra-low-power transceiver in a multi-transmitter and multi-receiver scenario," in IEEE Sensors, 2011, pp. 1205-1208.

[17] P. Christ, F. Werner, U. Rückert, and J. Mielebacher, "An approach for determining linear velocities of athletes from acceleration measurements using a neural network," in Proc. of the 6th Int. Conf. on Biomechanics, 2011, pp. 105-112.

[18] W. D. McArdle, F. I. Katch, and V. L. Katch, Exercise physiology: Nutrition, energy, and human performance, 7th ed. Lippincott Williams \& Wilkins, 2010.

[19] I. F. Zakeri, A. L. Adolph, M. R. Puyau, F. A. Vohra, and N. F. Butte, "Multivariate adaptive regression splines models for the prediction of energy expenditure in children and adolescents," J Appl Physiol, vol. 108, no. 1, pp. 128-136, 2010.

[20] A. Abraham and D. Steinberg, "Mars: Still an alien planet in soft computing?" in Computational Science - ICCS 2001, ser. Lecture Notes in Computer Science. Springer, 2001, vol. 2074, pp. 235-244.

[21] F. Conconi, M. Ferrari, P. G. Ziglio, P. Droghetti, and L. Codeca, "Determination of the anaerobic threshold by a noninvasive field test in runners." J Appl Physiol Respir Environ Exerc Physiol, vol. 52, no. 4, pp. 869-873, 1982.

[22] P. Rosskopf, Atemfrequenz als Parameter der Leistungdiagnostik und Trainingssteuerung : Untersuchung zur Anwendbarkeit im Breitensport. Kovač, 1996. 